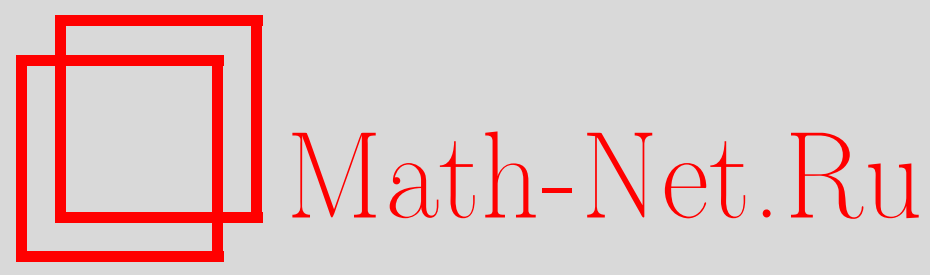

П. В. Ушаков, ІА-автоморфизмы метабелевых произведений двух абелевых групп, Матем. заметки, 2001, том 70, выпуск 3, 446-457

DOI: https://doi.org/10.4213/mzm756

Использование Общероссийского математического портала Math-Net.Ru подразумевает, что вы прочитали и согласны с пользовательским соглашением http://www . mathnet.ru/rus/agreement

Параметры загрузки:

IP: 54.237 .59 .107

26 апреля 2023 г., 11:43:11

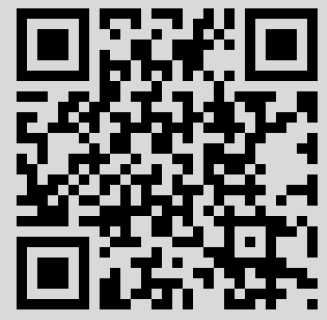




\section{ІА-АВТОМОРФИЗМЫ МЕТАБЕЛЕВЫХ ПРОИЗВЕДЕНИЙ ДВУХ АБЕЛЕВЫХ ГРУПП}

\section{П. В. Ушаков}

В этой статье предъявлены IA-автоморфизмы метабелевых произведений двух абелевых групп с кручением, которые не являются внутренними и, следовательно, не индуцируются никакими автоморфизмами свободных произведений тех же абелевых групп.

Библиограффия: 4 названия.

1. Введение. Автоморфизм группы $K$ назьвается IA- автоморфизмом, если он индуцирует тождественное отображение в факторгрупше $K / K^{\prime}$, где $K^{\prime}$ - коммутант $K$. Эндоморфизм групшы $K$ назьвается IA- эндоморфизмом, если он индуцирует тождественное отображение в факторгруппе $K / K^{\prime}$.

С. Бахмут, Е. Форманек, Х. Мочизуки [1] доказали, что если $F_{2}$ - абсолютно свободная группа ранга $2, R \triangleleft F_{2}, R \leqslant F_{2}^{\prime}$ и групшовое кольцо $\mathbb{Z}\left(F_{2} / R\right)$ без делителей нуля, то любой IА-автоморфизм группы $F_{2} / R^{\prime}$ внутренний.

Для любой группы $K$ мы обозначим через $\mathbb{Z} K$ целочисленноегруповое кольцо группы $K$, через $K^{\prime}$ мы обозначим коммутант группы $K$, а через $K^{\prime \prime}$ коммутант коммутанта группы $K$. В данной работе мы докажем следующие результаты.

Теорема 1. Пусть $A=A_{1} * A_{2}$ - свободное произведение двух нетривиальных конечных абелевых групп, не являюшихся одновременно ииклическими порядка 2. Допустим, что порядки групп $A_{1}$ и $A_{2}$ не являются взаимно простыми. Тогда не всякий IА-автоморфизм группы $A / A^{\prime \prime}$ является внутренним.

Теорема 2. Пусть $A=A_{1} * A_{2}$ - свободное произведение двух нетривиальных бесконечных абелевых групп. Если челочисленное групповое кольио $\mathbb{Z}\left(A_{1} \times A_{2}\right)$ прямого произведения групп $A_{1}$ и $A_{2}$ имеет нетривиальные единииы, то не всякий IA-автоморфизм группы $A / A^{\prime \prime}$ является внутренним.

Полезно отметить, что любой ІА-автоморфизм свободного произведения $A$ двух нетривиальных абелевых групп является внутренним; следовательно, невнутренний IAавтоморфизм группы $A / A^{\prime \prime}$ не индуцируется никаким IA-автоморфизмом групшы $A$.

В данной работе мы будем использовать следующие обозначения. Через $A=A_{1} * A_{2}$ мы обозначим свободное произведение двух нетривиальных абелевьх групा $A_{1}$ и $A_{2}$. Мы обозначим $G \stackrel{\text { def }}{=} A / A^{\prime \prime}, H \stackrel{\text { def }}{=} A / A^{\prime}$. Для каждого $x \in G$ мы обозначим его образ

Исследования частично поддержаны Российским фондом фундаментальных исследований, гранты № 96-01-00420, № 96-15-96050 и № 99-01-00894. 
в $H$ через $\bar{x}$. Для каждого $x \in A$ мы обозначим его образ в $H$ через $\bar{x}$. Для каждого $x \in A$ мы обозначим его образ в $G$ через $\tilde{x}$. Образы групп $A_{1}, A_{2}$ в групше $G$ мы обозначим через $G_{1}, G_{2}$ соответственно. Образы групा $A_{1}, A_{2}$ в группе $H$ мы обозначим через $H_{1}, H_{2}$ соответственно. Под $\mathbb{Z} H\left(H_{1}-1\right)$ мы будем понимать идеал кольца $\mathbb{Z} H$, порожденный всеми $(h-1), h \in H_{1}$. Аналогично, под $\mathbb{Z} H\left(H_{2}-1\right)$ мы будем понимать идеал кольца $\mathbb{Z} H$, порожденный всеми $(h-1), h \in H_{2}$.

2. Обобщенные дифференцирования групповых колец. ${ }^{1}$ Вложение Шмелькина (см. [2]) позволяет ввести отображения $\bar{D}_{1}, \bar{D}_{2}$, которые играют при исследовании группы $A / A^{\prime \prime}$ ту же роль, которую дифференцирования $\Phi$ окса $\bar{\partial} / \partial\left(x_{1}\right), \bar{\partial} / \partial\left(x_{2}\right)$ играют при исследовании групп вида $F_{2} / F_{2}^{\prime \prime}$, где $F_{2}=F_{2}\left(x_{1}, x_{2}\right)$ - свободная группа с двумя порождающими $x_{1}, x_{2}$.

Напомним формулировку вложения Шмелькина. Пусть $P \triangleleft A, P \subseteq C, C$ - декартова подгрупша группы $A$. Пусть $M_{P}-$ свободньй модуль с двумя свободными порождающими $s_{1, P}, s_{2, P}$ над $\mathbb{Z}(A / P)$. Пусть $\Xi_{P}-$ группа матриц вида

$$
\left\|\begin{array}{cc}
u & u_{1} \\
0 & 1
\end{array}\right\|, \quad u \in A / P, \quad u_{1} \in M_{P} .
$$

Тогда существует вложение $\chi_{P}: A / P^{\prime} \rightarrow \Xi_{P}$ такое, что

$$
\chi_{P}\left(g P^{\prime}\right)=\left\|\begin{array}{cc}
g P & (g P-1) s_{1, P} \\
0 & 1
\end{array}\right\|, \quad g \in A_{1}
$$

и

$$
\chi_{P}\left(g P^{\prime}\right)=\left\|\begin{array}{cc}
g P & (g P-1) s_{2, P} \\
0 & 1
\end{array}\right\|, \quad g \in A_{2} .
$$

ОПРЕДЕЛЕНИЕ. Обобщенным дифференцированием по компоненте $i=1,2$ называется отображение $D_{i}: \mathbb{Z} A \rightarrow \mathbb{Z} A$, удовлетворяющее следующим аксиомам:

1) для любых $a, b \in \mathbb{Z} A$ выполнено $D_{i}(a+b)=D_{i}(a)+D_{i}(b)$,

2) для любых $a, b \in \mathbb{Z} A$ выполнено $D_{i}(a b)=a D_{i}(b)+\varepsilon(b) D_{i}(a)$,

3) для любого $a \in A_{i}$ выполнено $D_{i}(a)=a-1$,

4) для любого $a \in A_{j}, j \neq i$, выполнено $D_{i}(a)=0$,

где $\varepsilon$ - гомоморфизм тривиализации (также называемый дополняющим гомоморфизмOM).

Покажем, что определение обобщенных дифференцирований корректно. Понятно, что если существует хотя бы одно отображение, удовлетворяющее свойствам 1)-4) определения дифференцирований, то оно единственно. Покажем, что одно такое отображение существует. Будем использовать вложение $\chi_{\{1\}}$. Мы имеем

$$
\chi_{\{1\}}(x)=\left\|\begin{array}{ll}
x & \hat{x} \\
0 & 1
\end{array}\right\|
$$

\footnotetext{
${ }^{1}$ Уже после того, как эта статья была отдана в редакцию, автор узнал, что подобное обобщение дифференцирований Фокса было введено несколькими годами ранее в работах Н. С. Романовского.
} 
для всех $x \in A$. Каждый элемент $r \in \mathbb{Z} A$ может быть представлен в виде суммы

$$
r=\sum_{i=1}^{I} z_{i} r_{i}, \quad 0 \neq z_{i} \in \mathbb{Z}, \quad r_{i} \in A, \quad r_{i} \neq r_{j}, \quad i \neq j .
$$

Рассмотрим отображение $D$, которое отображает $r$ в $\sum_{i=1}^{I} z_{i} \hat{r}_{i}$. Очевидно, что для всех $u, v \in A$ вьполнено $D(u v)=u D(v)+D(u)$. Более того, если $u \in A_{k}, k=1,2$, то $D(u)=(u-1) s_{k,\{1\}}$. Для всех $u, v \in \mathbb{Z} A$ выполнено $D(u+v)=D(u)+D(v)$. Пусть $r=\sum_{i=1}^{I} z_{i} r_{i} \in \mathbb{Z} A, p=\sum_{j=1}^{J} z_{j}^{\prime} r_{j}^{\prime} \in \mathbb{Z} A$. Тогда

$$
\begin{aligned}
D(r p) & \left.=D\left(\sum_{i=1, I ; j=1, J} z_{i} z_{j}^{\prime} r_{i} r_{j}^{\prime}\right) \quad \text { (по определению } D\right) \\
& =\sum_{i=1, I ; j=1, J} z_{i} z_{j}^{\prime} D\left(r_{i} r_{j}^{\prime}\right)=\sum_{i=1, I ; j=1, J} z_{i} z_{j}^{\prime}\left(r_{i} D\left(r_{j}^{\prime}\right)+D\left(r_{i}\right)\right) \\
& =\sum_{i=1, I ; j=1, J} z_{i} z_{j}^{\prime} r_{i} D\left(r_{j}^{\prime}\right)+\sum_{i=1, I ; j=1, J} z_{i} z_{j}^{\prime} D\left(r_{i}\right)=r D(p)+\varepsilon(p) D(r) .
\end{aligned}
$$

Рассмотрим отображение $\omega_{k}, k=1,2$, которое отображает $\sum_{j=1,2} a_{j} s_{j,\{1\}}, a_{j} \in \mathbb{Z} A$, в $a_{k}$. Очевидно, что отображение $\omega_{k}(D)$ существует, единственно и удовлетворяет свойствам 1)-4) определения дифференцирований. Следовательно, обобщенные дифференцирования определены корректно.

ОПРЕДЕЛЕНИЕ. Индуцированным обобщенным дифференцированием по компоненте $i=1,2$ назьвается отображение $\bar{D}_{i}: \mathbb{Z} A \rightarrow \mathbb{Z} H$, определяемое по правилу $\bar{D}_{i}(a) \stackrel{\text { def }}{=} \tau\left(D_{i}(a)\right), a \in A, \tau$ - естественное отображение $\mathbb{Z} A \rightarrow \mathbb{Z} H$.

Легко заметить, что

$$
\chi_{\{1\}}(x)=\left\|\begin{array}{cc}
x & D_{1}(x) s_{1,\{1\}}+D_{2}(x) s_{2,\{1\}} \\
0 & 1
\end{array}\right\|, \quad x \in A .
$$

Из этого и вложения $\chi_{A^{\prime}}$ мы видим, что $\bar{D}_{i}(a)=0, i=\overline{1,2}$, тогда и только тогда, когда $a \in A^{\prime \prime}$. Фактически, дифференцирования $\bar{D}_{i}, i=\overline{1,2}$, отображают $\mathbb{Z} G$ в $\mathbb{Z} H$, поскольку $\bar{D}_{i}(a b)=\bar{D}_{i}(a)$ для всяких $a \in A, b \in A^{\prime \prime}$.

Важнейшим свойством дифференцирований является следующее (см. [3]). Если $a \in$ $\mathbb{Z} H\left(H_{1}-1\right), b \in \mathbb{Z} H\left(H_{2}-1\right), u \in H, u-1=a+b$, то существует $v \in G, \bar{D}_{1}(v)=a$, $\bar{D}_{2}(v)=b, \bar{v}=u$. Обратно, для всякого $v \in G$ выполнено $\bar{D}_{1}(v) \in \mathbb{Z} H\left(H_{1}-1\right)$, $\bar{D}_{2}(v) \in \mathbb{Z} H\left(H_{2}-1\right), \bar{D}_{1}(v)+\bar{D}_{2}(v)=\bar{v}-1$.

3. Задание ІА-автоморфизмов группы $G$ матрицами над $\mathbb{Z} H$. Рассмотрим множество матриц $\widetilde{\mathrm{M}}_{2}(\mathbb{Z} H)$, состоящее из всех матриц

$$
\left\|\begin{array}{cc}
1+s_{1} & -s_{1} \\
-s_{2} & 1+s_{2}
\end{array}\right\|
$$

таких, что $s_{1} \in \mathbb{Z} H\left(H_{2}-1\right), s_{2} \in \mathbb{Z} H\left(H_{1}-1\right)$. Это множество замкнуто относительно операции умножения. Действительно, пусть

$$
M_{1}=\left\|\begin{array}{cc}
1+s_{1} & -s_{1} \\
-s_{2} & 1+s_{2}
\end{array}\right\| \in \widetilde{\mathrm{M}}_{2}(\mathbb{Z} H), \quad M_{2}=\left\|\begin{array}{cc}
1+c_{1} & -c_{1} \\
-c_{2} & 1+c_{2}
\end{array}\right\| \in \widetilde{\mathrm{M}}_{2}(\mathbb{Z} H) .
$$


Тогда

$$
\begin{aligned}
& \left.M_{1} M_{2}=\| \begin{array}{cc}
1+\left(s_{1}+c_{1}+s_{1} c_{1}+s_{1} c_{2}\right) & -\left(s_{1}+c_{1}+s_{1} c_{1}+s_{1} c_{2}\right) \\
-\left(s_{2}+s_{2} c_{1}+c_{2}+s_{2} c_{2}\right) & 1+\left(s_{2}+s_{2} c_{1}+c_{2}+s_{2} c_{2}\right)
\end{array}\right) \|, \\
& \left(s_{1}+c_{1}+s_{1} c_{1}+s_{1} c_{2}\right) \in \mathbb{Z} H\left(H_{2}-1\right), \quad\left(s_{2}+s_{2} c_{1}+c_{2}+s_{2} c_{2}\right) \in \mathbb{Z} H\left(H_{1}-1\right) .
\end{aligned}
$$

Каждой матрице

$$
M=\left\|\begin{array}{cc}
1+s_{1} & -s_{1} \\
-s_{2} & 1+s_{2}
\end{array}\right\|
$$

из $\widetilde{\mathrm{M}}_{2}(\mathbb{Z} H)$ мы сопоставим отображение $\alpha_{M}$, определяемое по правилу

$$
\alpha_{M}: u \rightarrow v, \quad u, v \in G, \quad\left(\bar{D}_{1}(v), \bar{D}_{2}(v)\right)=\left(\bar{D}_{1}(u), \bar{D}_{2}(u)\right)\left\|\begin{array}{cc}
1+s_{1} & -s_{1} \\
-s_{2} & 1+s_{2}
\end{array}\right\|
$$

Отображение $\alpha_{M}$ определено корректно, поскольку

$$
\begin{gathered}
\bar{D}_{1}(u)\left(1+s_{1}\right)-\bar{D}_{2}(u) s_{2} \in \mathbb{Z} H\left(H_{1}-1\right), \\
-\bar{D}_{1}(u)\left(s_{1}\right)+\bar{D}_{2}(u)\left(1+s_{2}\right) \in \mathbb{Z} H\left(H_{2}-1\right) \\
\bar{D}_{1}(u)\left(1+s_{1}\right)-\bar{D}_{2}(u) s_{2}-\bar{D}_{1}(u)\left(s_{1}\right)+\bar{D}_{2}(u)\left(1+s_{2}\right)=\bar{D}_{1}(u)+\bar{D}_{2}(u)=\bar{u}-1
\end{gathered}
$$

откуда следует, что каждому $u \in G$ сопоставляется, и единственный, $v \in G$.

Проверим, что $\alpha_{M}$ является эндоморфизмомгруппы $G$. Допустим, $u_{1}, u_{2} \in G$. Пусть $\alpha_{M}\left(u_{1}\right)=v_{1}, \alpha_{M}\left(u_{2}\right)=v_{2}$. Докажем, что $v_{1} v_{2}=\alpha_{M}\left(u_{1} u_{2}\right)$. Заметим, что $\bar{u}_{1}=\bar{v}_{1}$,

$$
\begin{aligned}
\bar{D}_{1}\left(v_{1} v_{2}\right) & =\bar{u}_{1} \bar{D}_{1}\left(v_{2}\right)+\bar{D}_{1}\left(v_{1}\right) \\
& =\bar{u}_{1}\left(\bar{D}_{1}\left(u_{2}\right)\left(1+s_{1}\right)-\bar{D}_{2}\left(u_{2}\right) s_{2}\right)+\bar{D}_{1}\left(u_{1}\right)\left(1+s_{1}\right)-\bar{D}_{2}\left(u_{1}\right) s_{2} \\
& =\left(\bar{u}_{1} \bar{D}_{1}\left(u_{2}\right)+\bar{D}_{1}\left(u_{1}\right)\right)\left(1+s_{1}\right)-\left(\bar{u}_{1} \bar{D}_{2}\left(u_{2}\right)+\bar{D}_{2}\left(u_{1}\right)\right) s_{2} \\
& =\bar{D}_{1}\left(u_{1} u_{2}\right)\left(1+s_{1}\right)-\bar{D}_{2}\left(u_{1} u_{2}\right) s_{2} .
\end{aligned}
$$

Аналогично,

$$
\bar{D}_{2}\left(v_{1} v_{2}\right)=-\bar{D}_{1}\left(u_{1} u_{2}\right) s_{1}+\bar{D}_{2}\left(u_{1} u_{2}\right)\left(1+s_{2}\right)
$$

Следовательно, $v_{1} v_{2}=\alpha_{M}\left(u_{1} u_{2}\right)$.

Обратим внимание на то, что $\alpha_{M} \in \operatorname{IEnd}\left(\underset{\widetilde{M}}{G}, M \in \widetilde{\mathrm{M}}_{2}(\mathbb{Z} H)\right.$, поскольку $\overline{\alpha_{M}(u)}=\bar{u}$, $u \in G$. Заметим также, что отображение $\alpha: \widetilde{\mathrm{M}}_{2}(\mathbb{Z} H) \rightarrow \operatorname{IEnd}(G), M \rightarrow \alpha_{M}$, является гомоморфизмом, поскольку произведению матриц $M_{1} M_{2}$ соответствует композиция $\alpha_{M_{2}}\left(\alpha_{M_{1}}\right)$. Нам понадобится следующий результат.

ЛЕмма 1. Пусть $\beta \in \operatorname{Inn} G, u \in G, \beta: g \rightarrow$ ugu ${ }^{-1}, g \in G$. Cyществует $M \in \widetilde{\mathrm{M}}_{2}(\mathbb{Z} H)$ такая, что $\alpha_{M}=\beta$. При этом $\operatorname{det} M=\bar{u}$. 
ДокАЗАТЕЛьСТво. Покажем, что матрица

$$
M=\left\|\begin{array}{cc}
1+\bar{D}_{2}(u) & -\bar{D}_{2}(u) \\
-\bar{D}_{1}(u) & 1+\bar{D}_{1}(u)
\end{array}\right\| \in \widetilde{\mathrm{M}}_{2}(\mathbb{Z} H)
$$

может быть взята в качестве искомой. Достаточно проверить, что $\alpha_{M}(g)=\beta(g)$ для всех $g \in G_{1}$ и $g \in G_{2}$. Пусть $g \in G_{1}$. Тогда

$$
\begin{aligned}
\bar{D}_{2}\left(u g u^{-1}\right) & =\bar{u} \bar{D}_{2}\left(g u^{-1}\right)+\bar{D}_{2}(u)=\bar{u} \bar{g} \bar{D}_{2}\left(u^{-1}\right)+\bar{u} D_{2}(g)+\bar{D}_{2}(u) \\
& =-\bar{u} \bar{g} \bar{u}^{-1} \bar{D}_{2}(u)+\bar{D}_{2}(u)=(1-\bar{g}) \bar{D}_{2}(u) .
\end{aligned}
$$

Кроме того,

$$
\bar{D}_{1}\left(u g u^{-1}\right)=\overline{u g u^{-1}}-1-(1-\bar{g}) \bar{D}_{2}(u)=(\bar{g}-1)\left(\bar{D}_{2}(u)+1\right) .
$$

Следовательно, $\alpha_{M}(g)=\beta(g)$. Аналогичные рассмотрения можно провести для $g \in G_{2}$.

Tо, что $\operatorname{det} M=\bar{u}$, проверяется непосредственно. Лемма доказана.

Полезно отметить и то, что если $M \in \widetilde{\mathrm{M}}_{2}(\mathbb{Z} H), M \in \mathrm{GL}_{2}(\mathbb{Z} H)$, то $M^{-1} \in \widetilde{\mathrm{M}}_{2}(\mathbb{Z} H)$. Действительно, кольцо $\mathbb{Z} H$ коммутативно; следовательно, определитель матрицы $M$ существует и обратим. Пусть

$$
M=\left\|\begin{array}{cc}
1+s_{1} & -s_{1} \\
-s_{2} & 1+s_{2}
\end{array}\right\| \in \widetilde{M}_{2}(\mathbb{Z} H)
$$

Тогда $\operatorname{det} M=1+s_{1}+s_{2}$ и

$$
M^{-1}=\left\|\begin{array}{cc}
1-s_{1}(\operatorname{det} M)^{-1} & s_{1}(\operatorname{det} M)^{-1} \\
s_{2}(\operatorname{det} M)^{-1} & 1-s_{2}(\operatorname{det} M)^{-1}
\end{array}\right\| \in \widetilde{\mathrm{M}}_{2}(\mathbb{Z} H) .
$$

Продемонстрируем, как по матрище $M \in \widetilde{\mathrm{M}}_{2}(\mathbb{Z} H)$ строить явно $\alpha_{M}$.

Лемма 2. Допустим, что

$$
M=\left\|\begin{array}{cc}
1+a & -a \\
-b & 1+b
\end{array}\right\| \in \widetilde{\mathrm{M}}_{2}(\mathbb{Z} H)
$$

Предположим, что

$$
\begin{gathered}
a=\sum_{i=1}^{I} z_{i} \bar{a}_{i}\left(\bar{b}_{i}-1\right), \quad b=\sum_{j=1}^{J} t_{j} \bar{c}_{j}\left(\bar{d}_{j}-1\right), \\
I, J \in \mathbb{N} \cup\{0\}, \quad a_{i}, d_{j} \in A_{1}, \quad b_{i}, c_{j} \in A_{2}, \quad z_{i}, t_{j}= \pm 1 .
\end{gathered}
$$

(Очевидно, что всякие $a \in \mathbb{Z} H\left(H_{2}-1\right), b \in \mathbb{Z} H\left(H_{1}-1\right)$ могут быть записань в таком виде.) Тогда

$$
\alpha_{M}: g \rightarrow \begin{cases}g \prod_{i=1}^{I}\left[g, \tilde{b}_{i}^{-1} \tilde{a}_{i}^{-1}\right]^{z_{i}}, & g \in G_{1}, \\ g \prod_{j=1}^{J}\left[g, \tilde{d}_{j}^{-1} \tilde{c}_{i}^{-1}\right]^{t_{j}}, & g \in G_{2} .\end{cases}
$$


ДокАЗАТЕЛЬСтво. Проверим справедливость утверждений леммы непосредственно. Пусть $g \in G_{1}$. Тогда

$$
\bar{D}_{2}\left(g \prod_{i=1}^{I}\left[g, \tilde{b}_{i}^{-1} \tilde{a}_{i}^{-1}\right]^{z_{i}}\right)=\bar{g} \bar{D}_{2}\left(\prod_{i=1}^{I}\left[g, \tilde{b}_{i}^{-1} \tilde{a}_{i}^{-1}\right]^{z_{i}}\right)=\bar{g}\left(\sum_{i=1}^{I} z_{i} \bar{D}_{2}\left(\left[g, \tilde{b}_{i}^{-1} \tilde{a}_{i}^{-1}\right]\right)\right) .
$$

С другой стороны,

$$
\begin{aligned}
\bar{D}_{2}\left(\left[g, \tilde{b}_{i}^{-1} \tilde{a}_{i}^{-1}\right]\right) & =\bar{D}_{2}\left(g^{-1} \tilde{a}_{i} \tilde{b}_{i} g \tilde{b}_{i}^{-1} \tilde{a}_{i}^{-1}\right)=\bar{g}^{-1} \bar{D}_{2}\left(\tilde{a}_{i} \tilde{b}_{i} g \tilde{b}_{i}^{-1} \tilde{a}_{i}^{-1}\right) \\
& =\bar{g}^{-1} \bar{a}_{i} \bar{b}_{i} \bar{D}_{2}\left(g \tilde{b}_{i}^{-1} \tilde{a}_{i}^{-1}\right)+\bar{g}^{-1} \bar{D}_{2}\left(\tilde{a}_{i} \tilde{b}_{i}\right) \\
& =\bar{g}^{-1} \bar{a}_{i} \bar{b}_{i} \bar{g} \bar{D}_{2}\left(\tilde{b}_{i}^{-1} \tilde{a}_{i}^{-1}\right)+\bar{g}^{-1} \bar{D}_{2}\left(\tilde{a}_{i} \tilde{b}_{i}\right)=\left(\bar{g}^{-1}-1\right) \bar{D}_{2}\left(\tilde{a}_{i} \tilde{b}_{i}\right) \\
& =\left(\bar{g}^{-1}-1\right) \bar{a}_{i} \bar{D}_{2}\left(\tilde{b}_{i}\right)=\left(\bar{g}^{-1}-1\right) \bar{a}_{i}\left(\bar{b}_{i}-1\right) .
\end{aligned}
$$

Следовательно,

$$
\bar{D}_{2}\left(g \prod_{i=1}^{I}\left[g, \tilde{b}_{i}^{-1} \tilde{a}_{i}^{-1}\right]^{z_{i}}\right)=-(\bar{g}-1) \sum_{i=1}^{I} z_{i} \bar{a}_{i}\left(\bar{b}_{i}-1\right)=\bar{D}_{2}\left(\alpha_{M}(g)\right) .
$$

Поскольку

$$
\overline{g \prod_{i=1}^{I}\left[g, \tilde{b}_{i}^{-1} \tilde{a}_{i}^{-1}\right]^{z_{i}}}=\bar{g}
$$

мы имеем

$$
\bar{D}_{1}\left(\alpha_{M}(g)\right)=\bar{D}_{1}\left(g \prod_{i=1}^{I}\left[g, \tilde{b}_{i}^{-1} \tilde{a}_{i}^{-1}\right]^{z_{i}}\right)
$$

откуда вытекает

$$
\alpha_{M}(g)=g \prod_{i=1}^{I}\left[g, \tilde{b}_{i}^{-1} \tilde{a}_{i}^{-1}\right]^{z_{i}} .
$$

Аналогично, при $g \in G_{2}$ справедливо

$$
\alpha_{M}(g)=g \prod_{j=1}^{J}\left[g, \tilde{d}_{j}^{-1} \tilde{c}_{i}^{-1}\right]^{t_{j}}
$$

Лемма доказана.

\section{4. Метабелево произведение бесконечных абелевых групп.}

Лемма 3. Предположим, что $A_{1}, A_{2}$ - бесконечные абелевь группь. Допустим, что $\alpha_{M_{1}}=\alpha_{M_{2}}$ для некоторых $M_{1}, M_{2} \in \widetilde{\mathrm{M}}_{2}(\mathbb{Z} H)$. Тогда $\mathrm{M}_{1}=M_{2}$. 
ДоКАЗАТЕЛЬСТво. ДоПустим, что

$$
M_{1}=\left\|\begin{array}{cc}
1+s_{1} & -s_{1} \\
-s_{2} & 1+s_{2}
\end{array}\right\|, \quad M_{2}=\left\|\begin{array}{cc}
1+c_{1} & -c_{1} \\
-c_{2} & 1+c_{2}
\end{array}\right\| .
$$

Для каждого $g \in G_{1}$ выполнено $\bar{D}_{1}(g)=\bar{g}-1, \bar{D}_{2}(g)=0$. Поскольку для всех $g \in G_{1}$ выполнено $\alpha_{M_{1}}(g)=\alpha_{M_{2}}(g)$, мы имеем $(h-1)\left(s_{1}-c_{1}\right)=0$ для всех $h \in H_{1}$. Ввиду бесконечности подгруппы $H_{1}$ получим, что $s_{1}=c_{1}$. Аналогично, ввиду бесконечности $H_{2}$ имеем $s_{2}=c_{2}$. Лемма доказана.

Лемма 4. Пусть $a \in \mathbb{Z} H, \varepsilon(a)=1$, где $\varepsilon-$ гомоморфизм тривиализачии. Тогда $a=1+b+c$, әде $b \in \mathbb{Z} H\left(H_{2}-1\right), c \in \mathbb{Z} H\left(H_{1}-1\right)$.

ДоказАтельство. Мы можем записать $a=1+d, \varepsilon(d)=0$. Далее, $d=\sum_{i=1}^{I} \varepsilon_{i} u_{i} v_{i}$, $u_{i} \in H_{1}, v_{i} \in H_{2}, \varepsilon_{i}= \pm 1, I \in \mathbb{N} \cup\{0\}$. Очевидно, что

$$
\sum_{i=1}^{I} \varepsilon_{i} u_{i} v_{i}=\sum_{i=1}^{I} \varepsilon_{i} u_{i}\left(v_{i}-1\right)+\sum_{i=1}^{I} \varepsilon_{i} u_{i}
$$

Легко видеть, что $\varepsilon\left(\sum_{i=1}^{I} \varepsilon_{i} u_{i}\right)=0$, откуда следует, что $\sum_{i=1}^{I} \varepsilon_{i} u_{i} \in \mathbb{Z} H\left(H_{1}-1\right)$. Возьмем $b=\sum_{i=1}^{I} \varepsilon_{i} u_{i}\left(v_{i}-1\right)$ и $c=\sum_{i=1}^{I} \varepsilon_{i}\left(u_{i}-1\right)=\sum_{i=1}^{I} \varepsilon_{i} u_{i}$. Они удовлетворяют формулировке леммы. Лемма доказана.

ДоКАЗАТЕЛЬСТво тЕОРемЫ 2. Допустим, что $\mathbb{Z} H$ имеет некоторую нетривиальную единицу $u \in \mathbb{Z} H$. Без ограничения общности можно предположить, что $\varepsilon(u)=1$. Тогда по лемме 4 существуют $b \in \mathbb{Z} H\left(H_{1}-1\right), a \in \mathbb{Z} H\left(H_{2}-1\right)$ такие, что $u=1+a+b$. Возьмем в качестве искомого автоморфизма

$$
\alpha_{M}, M=\left\|\begin{array}{cc}
1+a & -a \\
-b & 1+b
\end{array}\right\| \in \widetilde{\mathrm{M}}_{2}(\mathbb{Z} H) .
$$

Эндоморфизм $\alpha_{M}$ является автоморфизмом, поскольку $u$ - обратимьй элемент кольца $\mathbb{Z} H$. С другой стороны, если бы этот автоморфизм являлся внутренним, то по лемме 1 этот автоморфизм задавался бы матрицей $M^{\prime} \in \widetilde{\mathrm{M}}_{2}(\mathbb{Z} H), \operatorname{det} M^{\prime} \in H$. По лемме 3 $M=M^{\prime}$. Но это противоречит выбору элемента $u$. Теорема доказана.

Отметим, что "почти каждое" кольцо $\mathbb{Z} H$ с элементами конечного порядка содержит нетривиальные единицы. В действительности, Г. Хигман [4] доказал, что если $K$ - конечная абелева групша, то $\mathbb{Z} K$ не содержит нетривиальных единиц тогда и только тогда, когда либо $K$-прямоепроизведение нескольких циклических групп порядков 2 и 3 , либо $K$ - прямое произведение нескольких циклических групп порядков 2 и 4.

Завершим раздел примером. Пусть $A_{2}, A_{1}$ - бесконечные абелевы групшы. Допустим, что $t \in A_{1}, t^{5}=1$. Тогда

$$
\left(1-t+t^{2}\right)\left(t+t^{2}-t^{4}\right)=t-t^{2}+t^{3}+t^{2}-t^{3}+t^{4}-t^{4}+t^{5}-t^{6}=t^{5}=1 .
$$

Построим автоморфизм, указанный в доказательстве теоремы 2. Положим

$$
M=\left\|\begin{array}{cc}
1 & 0 \\
(\bar{t}-1)-\left(\bar{t}^{2}-1\right) & 1+\left(\bar{t}^{2}-1\right)-(\bar{t}-1)
\end{array}\right\| .
$$


Тогда

$$
M^{-1}=\left\|\begin{array}{c}
1 \\
-(\bar{t}-1)-\left(\bar{t}^{2}-1\right)+\left(\bar{t}^{2}-1\right)
\end{array} \quad \begin{array}{c}
0 \\
1+(\bar{t}-1)+\left(\bar{t}^{2}-1\right)-\left(\bar{t}^{2}-1\right)
\end{array}\right\| .
$$

Имеем

$$
\alpha_{M}:\left\{\begin{array}{ll}
g \rightarrow g, & g \in G_{1}, \\
g \rightarrow g\left[g, \tilde{t}^{4}\right]^{-1}\left[g, \tilde{t}^{3}\right], & g \in G_{2},
\end{array} \quad \text { и } \quad \alpha_{M^{-1}}: \begin{cases}g \rightarrow g, & g \in G_{1}, \\
g \rightarrow g\left[g, \tilde{t}^{4}\right]\left[g, \tilde{t}^{3}\right][g, \tilde{t}]^{-1}, & g \in G_{2} .\end{cases}\right.
$$

5. Метабелево произведение конечных абелевых групп. В этом разделе мы докажем теорему 1 . Мы предполагаем, что $A_{1}, A_{2}$ - конечные абелевы групы и их порядки не являются взаимно простьми. Без ограничения общности мы можем предполагать, что $A_{1}=C \times D, A_{2}=E \times F$, где $C, E$ - конечные абелевы, а $D, F$-циклические группы не взаимно простых порядков.

Пусть $|C|=n_{1},|D|=n_{2},|E|=k_{1},|F|=k_{2},\left(k_{2}, n_{2}\right) \neq 1$.

Обозначим через $\mathscr{A}$ идеал кольца $\mathbb{Z} H$, порожденный элементом

$$
\dot{a}=\left(\sum_{a \in A_{1}} \bar{a}\right)=\left(\sum_{c \in C} \bar{c}\right)\left(\sum_{d \in D} \bar{d}\right) .
$$

Аналогично, обозначим через $\mathscr{B}$ идеал кольца $\mathbb{Z} H$, порожденньй элементом

$$
\hat{b}=\left(\sum_{b \in A_{2}} \bar{b}\right)=\left(\sum_{e \in E} \bar{e}\right)\left(\sum_{f \in F} \bar{f}\right) .
$$

Отметим, что если $a_{0} \in \mathbb{Z} H_{1}$ и для всех $a \in H_{1}$ вьполнено $(a-1) a_{0}=0$, то $a_{0}=l a ́$, $i \in \mathbb{Z}$. Аналогично, если $b_{0} \in \mathbb{Z} H_{2}$ и для всех $b \in H_{2}$ вьполнено $(b-1) b_{0}=0$, то $b_{0}=m \dot{m}$, $m \in \mathbb{Z}$.

Пусть $\mathscr{A}\left(H_{2}-1\right)=\mathscr{A} \cap \mathbb{Z} H\left(H_{2}-1\right), \mathscr{B}\left(H_{1}-1\right)=\mathscr{B} \cap \mathbb{Z} H\left(H_{1}-1\right)$. Обозначим также $\hat{a}=\left(\sum_{c \in C} \bar{c}\right)$ и $\hat{b}=\left(\sum_{e \in E} \bar{e}\right)$.

Матрицы из $\widetilde{\mathrm{M}}_{2}(\mathbb{Z} H)$ определяют IA-эндоморфизмы группы $G$. Справедлива

Лемма 5. Пусть

$$
\left\|\begin{array}{cc}
1-s_{1} & s_{1} \\
s_{2} & 1-s_{2}
\end{array}\right\|,\left\|\begin{array}{cc}
1-s_{1}^{\prime} & s_{1}^{\prime} \\
s_{2}^{\prime} & 1-s_{2}^{\prime}
\end{array}\right\| \in \widetilde{\mathrm{M}}_{2}(\mathbb{Z} H) .
$$

Эти матрицы определяют один и тот же ІА-эндоморфизм тогда и только тогда, когда $s_{1}-s_{1}^{\prime} \in \mathscr{A}\left(H_{2}-1\right), s_{2}-s_{2}^{\prime} \in \mathscr{B}\left(H_{1}-1\right)$.

ДокАЗАТЕЛЬСтво. Если $s_{1}-s_{1}^{\prime} \in \mathscr{A}\left(H_{2}-1\right)$ и $s_{2}-s_{2}^{\prime} \in \mathscr{B}\left(H_{1}-1\right)$, то для всякого $a \in H_{1}$ справедливо $(a-1) s_{1}=(a-1) s_{1}^{\prime}$ и для каждого $b \in H_{2}$ справедливо $(b-1) s_{2}=(b-1) s_{2}^{\prime}$, откуда следует, что матрицы определяют один и тот же IA-эндоморфизм.

Обратно, пусть матрицы определяют один и тот же IA-автоморфизм. Тогда для всех $a \in H_{1}$ справедливо $(a-1) s_{1}=(a-1) s_{1}^{\prime}$ и для каждого $b \in H_{2}$ справедливо $(b-1) s_{2}=(b-1) s_{2}^{\prime}$.

Очевидно, что

$$
\left(s_{1}-s_{1}^{\prime}\right)=h_{1}\left(g_{1}-1\right)+h_{2}\left(g_{2}-1\right)+\cdots+h_{m}\left(g_{m}-1\right), \quad g_{i} \in H_{2}, \quad h_{i} \in \mathbb{Z} H_{1} .
$$

Из того, что $(a-1)\left(s_{1}-s_{1}^{\prime}\right)=0$ для всех $a \in H_{1}$, следует, что $(a-1) h_{i}=0, i=\overline{1, m}$,

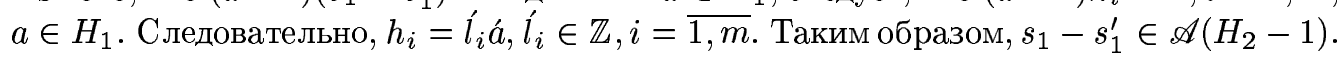
Аналогично, $s_{2}-s_{2}^{\prime} \in \mathscr{B}\left(H_{1}-1\right)$. Лемма доказана. 
ЛЕмма 6. Предположим, что матрицы

$$
K_{1}=\left\|\begin{array}{cc}
1-s_{1} & s_{1} \\
s_{2} & 1-s_{2}
\end{array}\right\| \quad u \quad K_{2}=\left\|\begin{array}{cc}
1-c_{1} & c_{1} \\
c_{2} & 1-c_{2}
\end{array}\right\|
$$

принадлежсат множеству $\widetilde{\mathrm{M}}_{2}(\mathbb{Z} H)$, причем

$$
K_{1} K_{2}=\left\|\begin{array}{cc}
1-d_{1} & d_{1} \\
d_{2} & 1-d_{2}
\end{array}\right\|
$$

əде $d_{1} \in \mathscr{A}\left(H_{2}-1\right), d_{2} \in \mathscr{B}\left(H_{1}-1\right), u$

$$
K_{2} K_{1}=\left\|\begin{array}{cc}
1-d_{3} & d_{3} \\
d_{4} & 1-d_{4}
\end{array}\right\|
$$

где $d_{3} \in \mathscr{A}\left(H_{2}-1\right), d_{4} \in \mathscr{B}\left(H_{1}-1\right)$. Тогда определяемые матрииами $K_{1}, K_{2}$ IA-эндоморфизмы $\alpha_{K_{1}}$ и $\alpha_{K_{2}}$ являются взаимнообратными IA-автоморфизмами.

ДокАЗАТЕльство. Эта лемма является непосредственным следствием леммы 5.

Обозначим

$$
r \stackrel{\text { def }}{=} \hat{a} \hat{b}-k_{2} \hat{a} \hat{b} \in \mathbb{Z} H\left(H_{2}-1\right), \quad p \stackrel{\text { def }}{=} \hat{a} \hat{b}-n_{2} \hat{a} \hat{b} \in \mathbb{Z} H\left(H_{1}-1\right) .
$$

Ввиду того, что

$$
\hat{a} \hat{b} \hat{a} \hat{b}=n_{1} k_{1} k_{2} \hat{a} \hat{b}, \quad \hat{a} \hat{b} \hat{a} \hat{b}=n_{1} k_{1} \hat{a} \hat{b}, \quad \hat{a} \hat{b} \hat{a} \hat{b}=n_{1} k_{1} \hat{a} \hat{b}, \quad a^{\prime} \hat{b} \hat{a} \hat{b}=n_{1} k_{1} \dot{a}^{\hat{b}}
$$

справедливы следующие соотношения:

$$
\begin{aligned}
r r=-n_{1} k_{1} k_{2} r, \quad p p & =-n_{1} k_{1} n_{2} p, \quad r p=-n_{1} k_{1} n_{2} r+\theta_{1}, \quad \theta_{1} \in \mathscr{A}\left(H_{2}-1\right), \\
p r & =-n_{1} k_{1} k_{2} p+\theta_{2}, \quad \theta_{2} \in \mathscr{B}\left(H_{1}-1\right) .
\end{aligned}
$$

Рассмотрим матрищы

$$
K_{1} \stackrel{\text { def }}{=}\left\|\begin{array}{cc}
1-m_{1} r & m_{1} r \\
l_{1} p & 1-l_{1} p
\end{array}\right\|, \quad K_{2} \stackrel{\text { def }}{=}\left\|\begin{array}{cc}
1-m_{2} r & m_{2} r \\
l_{2} p & 1-l_{2} p
\end{array}\right\|,
$$

где $m_{1}, m_{2}, l_{1}, l_{2} \in \mathbb{Z}$. Очевидно, $K_{1}, K_{2} \in \widetilde{\mathrm{M}}_{2}(\mathbb{Z} H)$. Покажем, что можно выбрать $m_{1}$, $m_{2}, l_{1}, l_{2}$ так, чтобы $K_{1} K_{2}$ и $K_{2} K_{1}$ удовлетворяли условиям леммы 6 . Получаем

$$
K_{1} K_{2}=\left\|\begin{array}{cc}
1-\left(m_{1} r+m_{2} r-m_{1} m_{2} r r-m_{1} l_{2} r p\right) & m_{1} r+m_{2} r-m_{1} m_{2} r r-m_{1} l_{2} r p \\
l_{1} p+l_{2} p-m_{2} l_{1} p r-l_{1} l_{2} p p & 1-\left(l_{1} p+l_{2} p-m_{2} l_{1} p r-l_{1} l_{2} p p\right)
\end{array}\right\| .
$$

Аналогично,

$$
K_{2} K_{1}=\left\|\begin{array}{cc}
1-\left(m_{2} r+m_{1} r-m_{2} m_{1} r r-m_{2} l_{1} r p\right) & m_{2} r+m_{1} r-m_{2} m_{1} r r-m_{2} l_{1} r p \\
l_{2} p+l_{1} p-m_{1} l_{2} p r-l_{2} l_{1} p p & 1-\left(l_{2} p+l_{1} p-m_{1} l_{2} p r-l_{2} l_{1} p p\right)
\end{array}\right\| .
$$


Положим

$$
\begin{aligned}
& \omega_{1} \stackrel{\text { def }}{=}\left(m_{1}+m_{2}+m_{1} m_{2} k_{2} n_{1} k_{1}+m_{1} l_{2} n_{2} n_{1} k_{1}\right) r, \\
& \omega_{2} \stackrel{\text { def }}{=}\left(l_{1}+l_{2}+m_{2} l_{1} k_{2} n_{1} k_{1}+l_{1} l_{2} n_{2} n_{1} k_{1}\right) p, \\
& \omega_{3} \stackrel{\text { def }}{=}\left(m_{2}+m_{1}+m_{2} m_{1} k_{2} n_{1} k_{1}+m_{2} l_{1} n_{2} n_{1} k_{1}\right) r, \\
& \omega_{4} \stackrel{\text { def }}{=}\left(l_{2}+l_{1}+m_{1} l_{2} k_{2} n_{1} k_{1}+l_{2} l_{1} n_{2} n_{1} k_{1}\right) p .
\end{aligned}
$$

Тогда получим

$$
K_{1} K_{2}=\left\|\begin{array}{cc}
1-\omega_{1}+\theta_{3} & \omega_{1}-\theta_{3} \\
\omega_{2}-\theta_{4} & 1-\omega_{2}+\theta_{4}
\end{array}\right\|, \quad \theta_{3} \in \mathscr{A}\left(H_{2}-1\right), \quad \theta_{4} \in \mathscr{B}\left(H_{1}-1\right),
$$

и

$$
K_{2} K_{1}=\left\|\begin{array}{cc}
1-\omega_{3}+\theta_{5} & \omega_{3}-\theta_{5} \\
\omega_{4}-\theta_{6} & 1-\omega_{4}+\theta_{6}
\end{array}\right\|, \quad \theta_{5} \in \mathscr{A}\left(H_{2}-1\right), \quad \theta_{6} \in \mathscr{B}\left(H_{1}-1\right) .
$$

Выберем

$$
m_{1} \stackrel{\text { def }}{=} \frac{n_{2}}{\left(n_{2}, k_{2}\right)}, \quad m_{2} \stackrel{\text { def }}{=}-\frac{n_{2}}{\left(n_{2}, k_{2}\right)}, \quad l_{1} \stackrel{\text { def }}{=}-\frac{k_{2}}{\left(n_{2}, k_{2}\right)}, \quad l_{2} \stackrel{\text { def }}{=} \frac{k_{2}}{\left(n_{2}, k_{2}\right)} .
$$

Тогда $l_{1}=-l_{2}, m_{2}=-m_{1}, m_{2} k_{2}=-l_{2} n_{2}, m_{1} k_{2}=-l_{1} n_{2}$. Таким образом, при данном выборе $l_{1}, l_{2}, m_{1}, m_{2}$ мы имеем $\omega_{1}=\omega_{2}=\omega_{3}=\omega_{4}=0$ и условия леммы 6 удовлетворены. Из этого следует, что $\alpha_{K_{1}}$ и $\alpha_{K_{2}}-\mathrm{IA-автоморфизмы,} \alpha_{K_{1}}=\alpha_{K_{2}}^{-1}$.

Лемма 7. Допустим, что $\left|A_{1}\right| \neq 2$ или $\left|A_{2}\right| \neq 2$. Тогда не существуют $a \in$ $\mathscr{A}\left(H_{2}-1\right), b \in \mathscr{B}\left(H_{1}-1\right), u \in H$ maкue, что $1-m_{1} r-l_{1} p+a+b=u$.

ДокАЗАТЕЛЬСТво. Предположим, что существуют $a, b, u$, указанные в формулировке леммы. По определению $r$ и $p$ мы имеем

$$
\begin{aligned}
1-m_{1} r-l_{1} p+a+b=1 & -m_{1} \hat{a} \hat{b}\left(\sum_{f \in F} \bar{f}-k_{2}\right)-l_{1} \hat{a} \hat{b}\left(\sum_{d \in D} \bar{d}-n_{2}\right) \\
& +\dot{a}\left(\sum_{i=1}^{d_{1}} \pm\left(f_{i}-1\right)\right)+\dot{b}\left(\sum_{j=1}^{d_{2}} \pm\left(e_{j}-1\right)\right),
\end{aligned}
$$

где $e_{j} \in H_{1}, f_{i} \in H_{2} ; d_{1}, d_{2} \in \mathbb{N}$. Рассмотрим гомоморфизм $\mathscr{P}: \mathbb{Z} H \rightarrow \mathbb{Z} H$, которьй отображает $\bar{a}_{i} \rightarrow 1, \bar{a}_{i} \in H_{1}$, и $\bar{b}_{j} \rightarrow \bar{b}_{j}, \bar{b}_{j} \in H_{2}$. Тогда

$$
\mathscr{P}(u)=\mathscr{P}\left(1-m_{1} r-l_{1} p+a+b\right)=1-m_{1} n_{1} \hat{b}\left(\sum_{f \in F} \bar{f}-k_{2}\right)+n_{1} n_{2}\left(\sum_{i=1}^{d_{1}} \pm\left(f_{i}-1\right)\right) .
$$

Заметим, что

$$
-m_{1} n_{1} \hat{b}\left(\sum_{f \in F} \bar{f}-k_{2}\right)+n_{1} n_{2}\left(\sum_{i=1}^{d_{1}} \pm\left(f_{i}-1\right)\right) \neq 0
$$


ввиду выбора $m_{1}$. Если $n_{1} \geqslant 1$, то

$$
\mathscr{P}(u)-1 \neq-m_{1} n_{1} \hat{b}\left(\sum_{f \in F} \bar{f}-k_{2}\right)+n_{1} n_{2}\left(\sum_{i=1}^{d_{1}} \pm\left(f_{i}-1\right)\right) .
$$

Следовательно, $n_{1}=1$. Аналогично, $k_{1}=1$. Мы видим, что $\hat{a}=1, \hat{b}=1$. Предположим, что $k_{2}>2$ и $F=\langle c\rangle_{k_{2}}$. Тогда

$$
-m_{1}\left(\sum_{f \in F} \bar{f}-k_{2}\right)+n_{2}\left(\sum_{i=1}^{d_{1}} \pm\left(f_{i}-1\right)\right)=\sum_{i=1}^{k_{2}-1} d_{i}^{\prime} \bar{c}^{i}+d^{\prime}, \quad 0 \neq d_{i}^{\prime} \in \mathbb{Z}, \quad d^{\prime} \in \mathbb{Z} .
$$

Если $d^{\prime} \neq 0$, то мощность носителя элемента

$$
-m_{1}\left(\sum_{f \in F} \bar{f}-k_{2}\right)+n_{2}\left(\sum_{i=1}^{d_{1}} \pm\left(f_{i}-1\right)\right)
$$

больше двух. Если $d^{\prime}=0$, то при некотором $1 \leqslant i \leqslant k_{2} \bar{c}^{i}=1$. И то, и другое невозможно. Лемма доказана.

ДоКАЗАТЕЛЬСТВо ТЕОРЕмЫ 1. В качестве автоморфизма, фигурирующего в формулировке данной теоремы, мы возьмем $\alpha_{K_{1}}$. Мы должны доказать, что этот автоморфизм не является внутренним. По лемме 1 если бы этот автоморфизм был внутренним, он бы задавался матрицей

$$
\left\|\begin{array}{cc}
1+\bar{D}_{2}(\bar{u}) & -\bar{D}_{2}(\bar{u}) \\
-\bar{D}_{1}(\bar{u}) & 1+\bar{D}_{1}(\bar{u})
\end{array}\right\|
$$

где $u \in G$ - элемент, при помоши которого $\alpha_{K_{1}}$ действовал бы сопряжением. Ввиду леммы 5 мы получим

$$
\bar{D}_{1}(\bar{u})=-l_{1} p+b, \quad b \in \mathscr{B}\left(H_{1}-1\right), \quad \bar{D}_{2}(\bar{u})=-m_{1} r+a, \quad a \in \mathscr{A}\left(H_{2}-1\right) .
$$

Поскольку $\bar{D}_{2}(\bar{u})+\bar{D}_{1}(\bar{u})=u-1$, мы имеем $-l_{1} p+b-m_{1} r+a+1=u$, что невозможно по лемме 7. Теорема доказана.

Необходимо отметить, что если $A_{1}=\mathbb{Z}_{2}=\langle a\rangle_{2}, A_{2}=\mathbb{Z}_{2}=\langle b\rangle_{2}$, то $A / A^{\prime \prime} \cong A$ и все автоморфизмы групшы $A / A^{\prime \prime}$ индуцируются автоморфизмами групшы $A$. ( $A^{\prime}$ является циклической подгруппой, порожденной $[a, b]$.)

Полезно заметить, что мы не требуем наличия у кольца $\mathbb{Z} H$ нетривиальных единиц. Например, в следующем примере $\mathbb{Z} H$ не имеет нетривиальных единиц (это следует из теоремы Хигмана), однако невнутренние IA-автоморфизмы имеются в наличии.

Возьмем $A_{1} \cong \mathbb{Z}_{2}, A_{1}=\left\langle a_{1}\right\rangle_{2}, A_{2}=\mathbb{Z}_{2} \times \mathbb{Z}_{2}, A_{2}=\left\langle b_{1}\right\rangle_{2} \times\left\langle b_{2}\right\rangle_{2}$. Мы имеем $\hat{a}=1$, $\hat{b}=\left(\bar{b}_{1}+1\right), r=\hat{b}\left(\bar{b}_{2}-1\right)=\left(\bar{b}_{1}+1\right)\left(\bar{b}_{2}-1\right), p=\hat{b}\left(\bar{a}_{1}-1\right)=\left(\bar{b}_{1}+1\right)\left(\bar{a}_{1}-1\right)$. При этом

$$
\begin{gathered}
K_{1}=\left\|\begin{array}{cc}
1-\left(\bar{b}_{1}+1\right)\left(\bar{b}_{2}-1\right) & \left(\bar{b}_{1}+1\right)\left(\bar{b}_{2}-1\right) \\
-\left(\bar{b}_{1}+1\right)\left(\bar{a}_{1}-1\right) & 1+\left(\bar{b}_{1}+1\right)\left(\bar{a}_{1}-1\right)
\end{array}\right\|, \\
K_{2}=\left\|\begin{array}{cc}
1+\left(\bar{b}_{1}+1\right)\left(\bar{b}_{2}-1\right) & -\left(\bar{b}_{1}+1\right)\left(\bar{b}_{2}-1\right) \\
\left(\bar{b}_{1}+1\right)\left(\bar{a}_{1}-1\right) & 1-\left(\bar{b}_{1}+1\right)\left(\bar{a}_{1}-1\right)
\end{array}\right\| .
\end{gathered}
$$


Соответственно получим

$$
\alpha_{K_{1}}: g \rightarrow \begin{cases}g\left[g, \tilde{b}_{1} \tilde{b}_{2}\right]^{-1}\left[g, \tilde{b}_{2}\right]^{-1}\left[g, \tilde{b}_{1}\right], & g \in G_{1}, \\ g\left[g, \tilde{a}_{1} \tilde{b}_{1}\right]\left[g, \tilde{a}_{1}\right], & g \in G_{2}\end{cases}
$$

и

$$
\alpha_{K_{2}}: g \rightarrow \begin{cases}g\left[g, \tilde{b}_{1} \tilde{b}_{2}\right]\left[g, \tilde{b}_{2}\right]\left[g, \tilde{b}_{1}\right]^{-1}, & g \in G_{1}, \\ g\left[g, \tilde{a}_{1} \tilde{b}_{1}\right]^{-1}\left[g, \tilde{a}_{1}\right]^{-1}, & g \in G_{2} .\end{cases}
$$

Автор благодарен А. Л. Шмелькину за поддержку и внимательное отношение к данной работе.

Автор признателен рецензенту за сделанные замечания.

\section{СПИСОК ЦИТИРОВАННОЙ ЛИТЕРАТУРЫ}

[1] Bachmuth S., Formanek E., Mochizuki H. Y. IA-automorphisms of certain two-generator torsion-free groups // J. Algebra. 1976. V. 40. P. 19-30.

[2] Шмелькин А. Л. О свободных произведениях групп // Матем. сб. 1969. Т. 79 (121). № 4 (8).

[3] Шмелькин А. Л. О некоторых факторгруппах свободного произведения // Тр. семинара им. И. Г. Петровского. 1979. Т. 5.

[4] Higman G. The units of group-rings // Proc. London Math. Soc. (2). 1940. V. 46. № 3. P. 231-248.

Московский государственный университет им. М.В. Ломоносова

Поступило

E-mail: pvushakov@mtu-net.ru

27.09.1999

Исправленный вариант

28.03.2001 